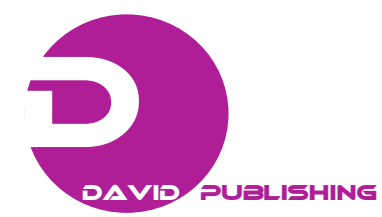

\title{
Earthquake-Caused Tsunami, Infection and Nuclear Plant Accident in Japan
}

\author{
Jun-ichi Sasaki \\ Department of Medical Technology, Hirosaki University School of Health Sciences, 2-7-1 Nishi-Aoyama, Morioka 020-0132, Japan \\ Corresponding author's e-mail: jnsasaki@tk2.so-net.ne.jp
}

\begin{abstract}
At 14:46 on March 11, 2011, Fukushima, Miyagi, Iwate Pref. and surrounding area were hit by the un-experienced huge earthquake with M9.1, followed by the deadly attack of tsunami waves with an estimated height of $34 \mathrm{~m}$. It devastated most of Northeastern coast and a number of victims involved deaths and missing counted over 25,000. As aftermath: 1) Infection of outbreaks of Noro virus, Legionella, Cl. Tetani, Rickettsia spp. were among the evacuated inhabitants in gymnasiums that were not under good living conditions; 2) High levels of arsenic contamination had been detected in mud washed ashore in coastal areas by tsunami. Quantities of arsenic exceeding government safety levels were detected in 36 out of 129 sampling points in the Northeast Tohoku region. 3) The most serious catastrophe due to tsunami was explosion of the Nuclear Power Plants in Fukushima that had scattered radioactive materials. Residents living within $20 \mathrm{~km}$ radius from the power plants explosion were evacuated to other areas. Residents living in the close area to the exploded power plant were exposed to radionuclides by inhalation or ingestion of contaminated air, drinking water, foods and foodstuffs. Some of the cattle were relocated to safer places to avoid contamination, and unmovable livestock and pets starved to death. Authors are still in the middle of tsunami's post-effects due to radioactive issues, piles of debris by tsunami, housing problem for evacuates, etc. The present situation of catastrophe and problems that are facing due to tsunami issues which could occur in any place at any time in the world will be focused in this manuscript.
\end{abstract}

Key words: Tsunami, earthquake, infection of outbreak, nuclear plant, explosion. 